\title{
Cystatin C in Evaluating Renal Function in Ureteral Calculi Hydronephrosis in Adults
}

\author{
Weipu Mao a,b Shenghua Liu ${ }^{b}$ Keyi Wang ${ }^{b}$ Miao Wang ${ }^{c}$ Heng Shi $^{b}$ \\ Qunlong Liu ${ }^{b}$ Meiyu Bao ${ }^{d}$ Bo Peng ${ }^{a}$ b Jiang Geng ${ }^{b}$ \\ ${ }^{a}$ Department of Urology, People's Hospital of Putuo District, Shanghai, China; ${ }^{\mathrm{b}}$ Department \\ of Urology, Shanghai Tenth People's Hospital, Tongji University, Shanghai, China; \\ 'Department of Orthopedics, Shanghai Tenth People's Hospital, School of Medicine, Tongji \\ University, Shanghai, China; 'Department of Central Laboratory, Shanghai Tenth People's \\ Hospital, Tongji University, Shanghai, China
}

\section{Keywords}

Cystatin C · Serum creatinine $\cdot$ Hydronephrosis $\cdot$ Renal function $\cdot$ Ureteral calculi

\begin{abstract}
Background: Serum cystatin C (CysC) is still becoming used as a marker of renal function but is far from being commonly used worldwide. The purpose of this study was to characterize the ureteral calculi patients with hydronephrosis-caused CysC changes in renal function. Methods: To better reflect the changes of renal function, we constructed models of ureteral obstruction in rats to mimic the hydronephrosis caused by human ureteral calculi. Moreover, our study included 200 patients diagnosed with ureteral calculi in our hospital between June 2017 and 2018. We compared the estimated glomerular filtration rate using different equations based on CysC and/or serum creatinine (SCr). Results: We found that the expression of Cys $\mathrm{C}$ and $\mathrm{SCr}$ increased with the prolonged obstruction time by enzyme linked immunosorbent assay. Moreover, quantitative real-time polymerase chain reaction, Western blot and immunohistochemistry further demonstrated that the expression of CysC increases with the degree of hydronephrosis. Among 200 patients with ureteral calculi, 40 (20.0\%) had no hydronephrosis, 110 (55.0\%) had mild hydronephrosis, 32 (16.0\%) had moderate hydronephrosis and $18(9.0 \%)$ had severe hydronephrosis. As the degree of hydronephrosis increased, the expression of neutrophil percentage, $\mathrm{Cys} C$, blood urea nitrogen, $\mathrm{SCr}$ and serum uric acid also
\end{abstract}

W.M., S.L., and K.W.: contributed equally to this work.

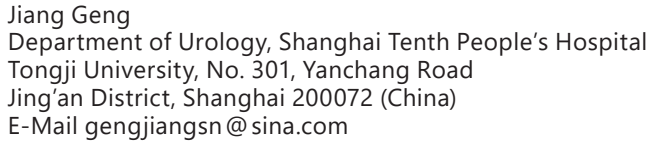

Bo Peng

Department of Urology, Shanghai Tenth People's Hospital School of Medicine, Tongji University, No. 301, Yanchang Road Jing'an District, Shanghai 200072 (China)

E-Mail pengbo6908@163.com 


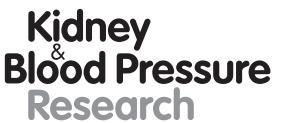

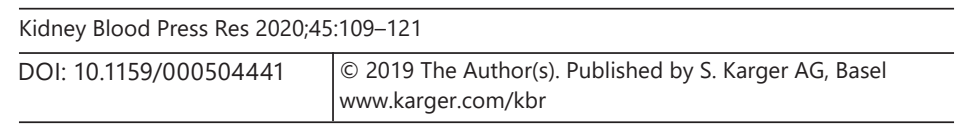

Mao et al.: CysC in Ureteral Calculi Hydronephrosis

increased. Multivariate analyses demonstrated that only Cys $C$ was an independent risk factor for hydronephrosis $(p=0.003)$. In addition, CysC and the Chronic Kidney Disease Epidemiology Collaboration (CKD-EPI) CysC equation showed the highest veracity in renal function estimation of patients with hydronephrosis caused by ureteral calculus. Conclusion: For patients with hydronephrosis caused by ureteral calculi, Cys $\mathrm{C}$ better reflects the changes in renal function, and the CKD-EPI CysC equation has the highest accuracy.

(C) 2019 The Author(s)

Published by S. Karger AG, Basel

\section{Introduction}

Ureteral calculi is a common condition in urology $[1,2]$, that is often secondary to intrarenal calculi [3]. Calculi can cause urinary tract obstruction, resulting in upper urinary tract dilatation, upper urinary tract infection, and the deterioration of renal function. The principle of treatment is to remove the upper urinary tract obstruction, relieve the upper urinary tract expansion and accumulation of urine, control the progress of infection, and then perform the treatment to remove the calculi [4].

Hydronephrosis is urine retention caused by the obstruction of various causes and is a common complication of urogenital system diseases. Due to the lack of symptoms in the early stages of the chronic hydronephrosis, some renal function has been damaged when symptoms appear [5]. Prolonged hydronephrosis causes increased intra-renal pressure, resulting in progressive dilatation of the renal pelvis and renal calyces and even atrophy of the renal parenchyma [6]. Therefore, it is particularly important to evaluate the degree of renal function impairment in hydronephrosis.

The glomerular filtration rate (GFR) is widely accepted as an ideal indicator for evaluating renal function $[7,8]$. Currently, the clearance of inulin is the gold standard used to estimate GFR. However, due to its complicated operation [9], blood urea nitrogen (BUN) $\beta_{2}-$ microglobulin $\left(\beta_{2}-\mathrm{MG}\right)$ and serum creatinine $(\mathrm{SCr})$ are often used to evaluate renal function clinically, but BUN, $\beta_{2}$-MG and SCr are often affected by other physiological and pathological factors and cannot reflect renal function accurately [10,11].

Cystatin $\mathrm{C}(\mathrm{CysC})$ is an endogenous cysteine protease inhibitor with a molecular weight of $13.3 \mathrm{kDa}$, and most nucleated cells are considered to be the source of secretion [12]. Normally, CysC is filtered through the glomerulus, reabsorbed and broken down into amino acids in the renal tubules. In recent years, serum CysC is a widely studied indicator of GFR. A large number of studies have shown that the metabolism of CysC is rarely affected by factors such as age, sex, weight, diet, infection and other factors, and has high specificity [13-15].

In this study, in order to better reflect the changes of renal function and the expression of CysC in kidney during hydronephrosis, we constructed models of ureteral obstruction in rats to mimic the hydronephrosis caused by human ureteral calculi. In addition, we analysed the patient's metabolic indicators and compared the estimated GFR (eGFR) using different equations based on $\mathrm{CysC}$ and/or SCr to provide clues for early detection of renal function changes in the presence of ureteral calculi.

\section{Materials and Methods}

Constructing Models of Ureteral Obstruction in Rats

Male SD rats (aged 10 weeks; body weight $300-400$ g, 3 rats per group) were purchased from Slaccas (Slaccas Laboratory Animal, Shanghai, China). The model of ureteral obstruction was constructed by ligating the unilateral ureter to simulate the change of hydronephrosis 


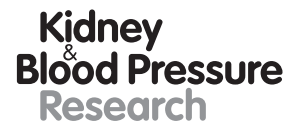

Kidney
Blood Pressure
Research \begin{tabular}{l|l}
\hline Kidney Blood Press Res 2020:45:109-121 \\
\hline DOI: 10.1159/000504441 & $\begin{array}{l}\text { @ 2019 The Author(s). Published by S. Karger AG, Basel } \\
\text { www.karger.com/kbr }\end{array}$ \\
\hline
\end{tabular}

Mao et al.: CysC in Ureteral Calculi Hydronephrosis

after human ureteral obstruction. Rats were treated with cervical dislocation at $0 \mathrm{~h}, 1$ day (D), 2 D, 3 D, 4 D, 5 D, 6 D, and 7 D after ligation. Bilateral kidney and cardiac blood were collected for the preparation of other experiments [16]. Animal research was conducted according to the ethical guidelines for animal experiment systems approved by the Animal Care and Use Committee of Tongji University.

\section{RNA Extraction and Quantitative Real-Time Polymerase Chain Reaction}

Total RNA was extracted from rat kidney tissues using Trizol reagent (Invitrogen, CA, USA) according to the manufacturer's protocol, and cDNA was generated using the cDNA synthesis kit (Takara Biotechnology, Dalian, China). Quantitative real-time polymerase chain reaction for CysC was performed using a SYBR Green PCR Kit (Takara Biotechnology, Dalian, China) with an ABI Prism 7500 Sequence Detection System (Applied Biosystems, Foster City, CA, USA). The primer sequences (Sango Biotech, China) were as follows: CysC forward, $5^{\prime}$-AACTACATGTAC-

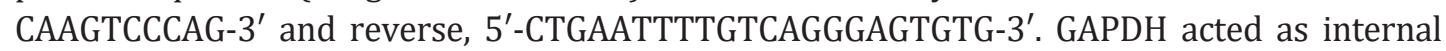
standards, and each sample was repeated 3 times. The relative quantification of expression of CysC was performed using the $2^{-\Delta \Delta \mathrm{Ct}}$ method and compared with internal standards.

\section{Enzyme Linked Immunosorbent Assay}

Cardiac blood obtained from each group of rats was centrifuged at 3,000 rpm for $10 \mathrm{~min}$. Then, the supernatant was collected and the levels of $\mathrm{CysC}$ and $\mathrm{SCr}$ in rat serum were detected according to the instructions of the enzyme linked immunosorbent assay kit (mlbio, Shanghai, China). The process was repeated 4 times for each sample.

\section{Western Blot}

Total protein was extracted from rat kidney tissues, separated using 10\% sodium dodecyl sulfate-polyacrylamide gel and imprinted on the nitrocellulose membrane (Sigma-Aldrich; Merck KGaA). Membranes were sealed at room temperature for $1 \mathrm{~h}$ with $5 \%$ non-fat milk and then incubated with primary antibody against CysC (Santa Cruz Biotechnology CA, USA; 1:200). GAPDH (Abcam, Cambridge, MA, USA) was used as an internal control. After incubation with the secondary antibody (Jackson Immno Research; 1:5,000) for $1 \mathrm{~h}$, the membranes were washed 3 times and the signals were visualized by the LI-COR Image Studio Lite imaging system.

\section{Haematoxylin-Eosin Staining and Immunohistochemistry}

The rat kidney tissues samples were fixed in 4\% paraformaldehyde at room temperature and then dehydrated via an ethanol solution and embedded in paraffin. Haematoxylin-eosin staining and immunohistochemistry were performed according to the previous procedure [17]. The sections were incubated with previous antibody for expression of CysC. The sections were imaged using the microscope (Leica Microsystems, Mannheim, Germany).

\section{Patients}

A total of 200 eligible patients diagnosed with ureteral calculi were identified between June 2017 and 2018. The inclusion criteria were as follows: patients with unilateral ureteral calculi diagnosed by clinical history, symptoms, signs, and confirmed by CT scan and patients who understood the contents of this study and voluntarily participated and accepted the test methods used in this study. The exclusion criteria were as follows: patients with a history of recurrent kidney stones or with stones in the kidney at the screening visit; patients with immune dysfunction, severe impairment of liver function, chronic renal insufficiency; malignant tumours, diabetes, coronary heart disease or any other known condition that might influence the CysC level; and hydronephrosis due to other causes. All patients were divided into the following 4 groups according to the Noble hydronephrosis method [18]: the no 


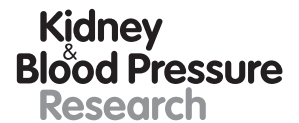

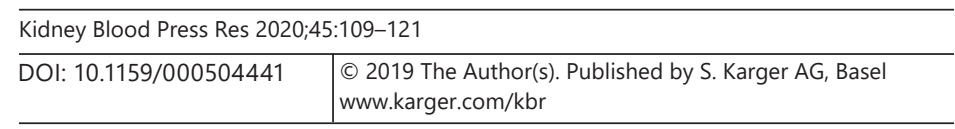

Mao et al.: CysC in Ureteral Calculi Hydronephrosis

hydronephrosis group, the mild hydronephrosis group, the moderate hydronephrosis group and the severe hydronephrosis group. The study protocol was approved by the Biomedical Ethics Committee of the Tenth Hospital in Shanghai, and written informed consent was obtained from all patients or their relatives.

\section{Serum Collection for All Patients}

Serum was collected in the morning, and all measurements were performed in the same laboratory. CysC was quantified using a fully automated particle-enhanced immunoturbidimetric assay with Sentinel Diagnostics reagents (Sentinel CH, Milan, Italy) on the Architect ci 16200 System (Abbott Laboratories, IL, USA) according to the manufacturer's instructions; (intra-assay CV $<1.5 \%$, inter-assay CV $<1 \%$ ). The baseline $\mathrm{SCr}$ was assessed in 200 participants and was analysed using an isotope dilution mass spectrometry traceable Jaffe kinetic assay for creatinine on a Hitachi 917 analyser (Roche Diagnostics GmbH, Mannheim Germany).

\section{Statistical Analysis}

To better evaluate changes in renal function, we used different GFR estimation equations such as the GFR, Cockcroft-Gault (CG) formula, modification of diet in renal disease (MDRD) equation, Chronic Kidney Disease Epidemiology Collaboration (CKD-EPI) CysC equation, CKD-EPI creatinine equation and CKD-EPI creatinine-CysC equation to calculate the eGFR [19-22].

Data was analysed using the SPSS software (version 20.0, SPSS, Inc., Chicago, IL, USA) and GraphPad Prism software (version 7.0, Inc., San Diego, CA, USA). Receiver operating characteristic curves were detected using MedCalc version 15.2.0 Software, which defined the sensitivity, specificity and differences in the area under the curve (AUC). A $p$ value $<0.05$ was considered significant. All continuous data is summarized as the means $\pm \mathrm{SD}$, and chi-square tests were performed to evaluate differences in categorical variables.

\section{Results}

The Expression of CysC and SCr Increased with the Prolonged Obstruction Time in Rats with Ureteral Obstruction

We constructed rat models of unilateral ureteral obstruction, after which we obtained kidney tissue and cardiac blood at different obstruction time points. Using enzyme-linked immunosorbent assay, we found that the concentration levels of CysC and SCr in the blood of rats increased with the prolonged obstruction time (Fig. 1a, b). In addition, we assessed the relative expression of CysC by quantitative real-time polymerase chain reaction in the kidney tissues of rats at different obstruction times, and our results showed that the expression of CysC increased with the prolonged obstruction time (Fig. 1c). Similarly, Immunohistochemistry also confirmed that CysC expression increased with time extension (Fig. 1d).

With the Extension of Obstruction Time, Hydronephrosis was Aggravated, Kidney Volume Increased and the Kidney Parenchyma Thickness Decreased

As seen by comparing the appearance of the kidneys and the transverse anatomy of the kidney, hydronephrosis increased with the extension of obstruction time, the kidney expanded in volume, and the renal parenchyma thickness decreased (Fig. 2a). Haematoxylin-eosin staining of the renal cortex and renal medulla revealed that the renal glomeruli atrophied, and the renal tubules dilatated in the cortex with the aggravation of hydronephrosis (Fig. 2b). Additionally, intramedullary renal interstitial oedema was increased, and a large number of inflammatory cells were infiltrated in the interstitium (Fig. 2c). 


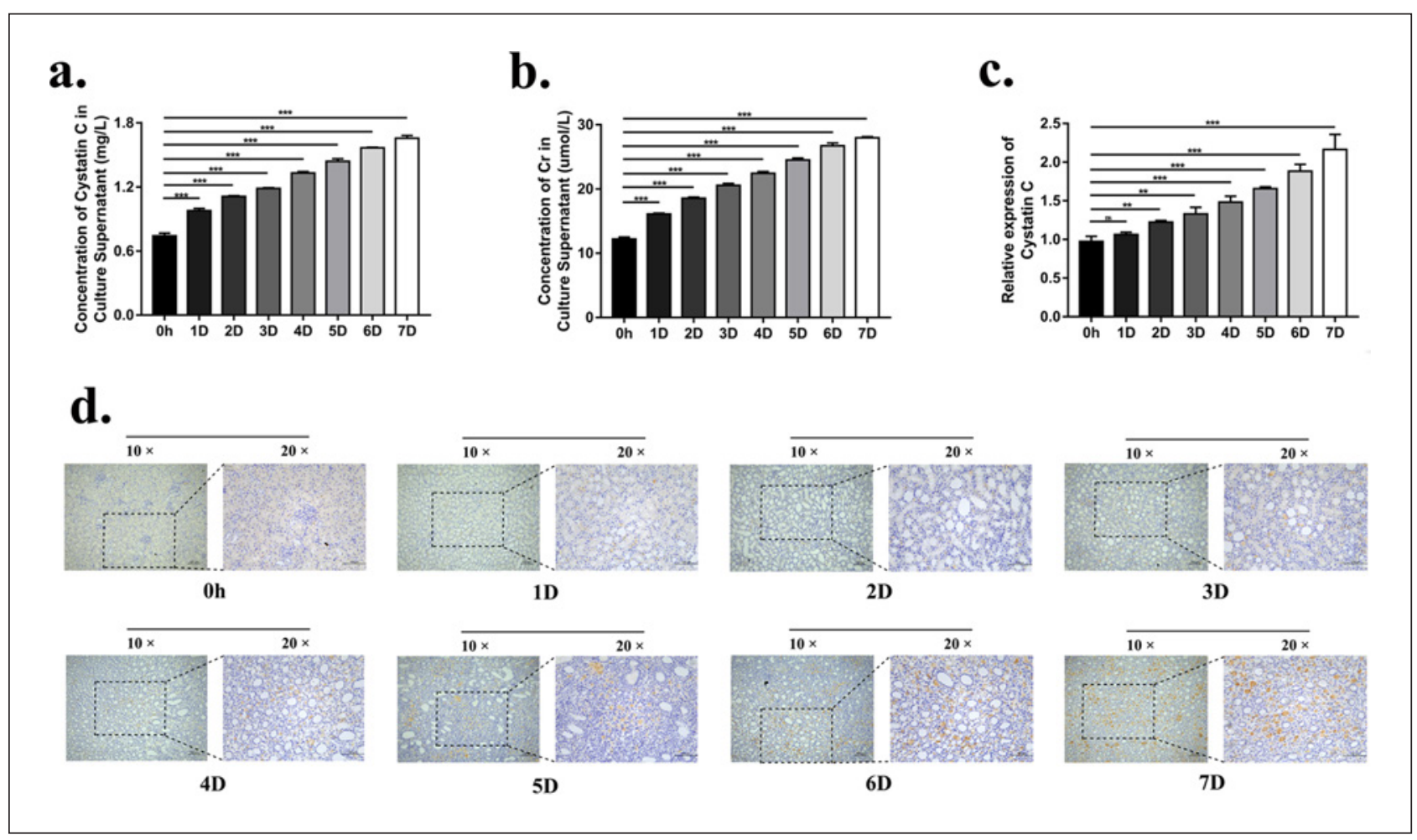

Fig. 1. The concentration of CysC and SCr increased with the increase in the degree of hydronephrosis in rats with ureteral obstruction; a The concentration of CysC was determined by ELISA ${ }^{* * *} p<0.001$; $\mathbf{b}$ The concentration of SCr was measured by ELISA ( ${ }^{* * *} p<0.001$ ); $\mathbf{c}$ The relative expression of CysC was detected by qRT-PCR ${ }^{* *} p<0.01,{ }^{* * *} p<0.001$ ); d IHC staining of CysC at different obstruction times; Scale bar $=200 \mu \mathrm{m}$ for $10 \times$ and $100 \mu \mathrm{m}$ for $20 \times$. CysC, Cystatin C; Cr, creatinine.

The Concentration of CysC and SCr Is Associated with the Degree of Hydronephrosis

Patients were divided into 4 groups according to the degree of hydronephrosis, of which $40(20.0 \%)$ patients had no hydronephrosis, 110 (55.0\%) had mild hydronephrosis, 32 $(16.0 \%)$ had moderate hydronephrosis, and 18 (9.0\%) had severe hydronephrosis. The variables associated with the degree of hydronephrosis, including the demographic and clinical characteristics, are described in Tables 1 and 2. Univariate analyses demonstrated that the degree of hydronephrosis was associated with the disease course, body weight, proteinuria, neutrophil percentage, CysC, BUN, SCr and serum uric acid (SUA; All $p<0.05$ ). The 200 ureteral calculi patients included in the study cohort had a median age of 52 years, median neutrophil percentage of $63.47 \%$, median CysC of $0.95 \mathrm{mg} / \mathrm{L}$, median BUN of $6.59 \mathrm{mmol} / \mathrm{L}$, median SCr of $102.88 \mu \mathrm{mol} / \mathrm{L}$ and median SUA of $342.02 \mu \mathrm{mol} / \mathrm{L}$. In addition, as the degree of hydronephrosis increased, the concentration of neutrophils, CysC, BUN, SCr and SUA also increased (Table 2). From Figure 3a and b, we could conclude that CysC has correlated between no hydronephrosis group and mild hydronephrosis group, while $\mathrm{SCr}$ has no correlation.

Stratified univariate and multivariate log regression were used to analyse the factors associated with hydronephrosis. As shown in Table 3, CysC and SCr were factors that affected hydronephrosis in the univariate log regression. However, the multivariate log regression model showed that only CysC (OR 6.28; 95\% CI 1.85-21.31; $p=0.003$ ) was an independent risk factor for hydronephrosis. 
Kidney

Blood Pressure

Research
Kidney Blood Press Res 2020;45:109-121

2019 The Author(s). Published by S. Karger AG, Basel www.karger.com/kbr

Mao et al.: CysC in Ureteral Calculi Hydronephrosis

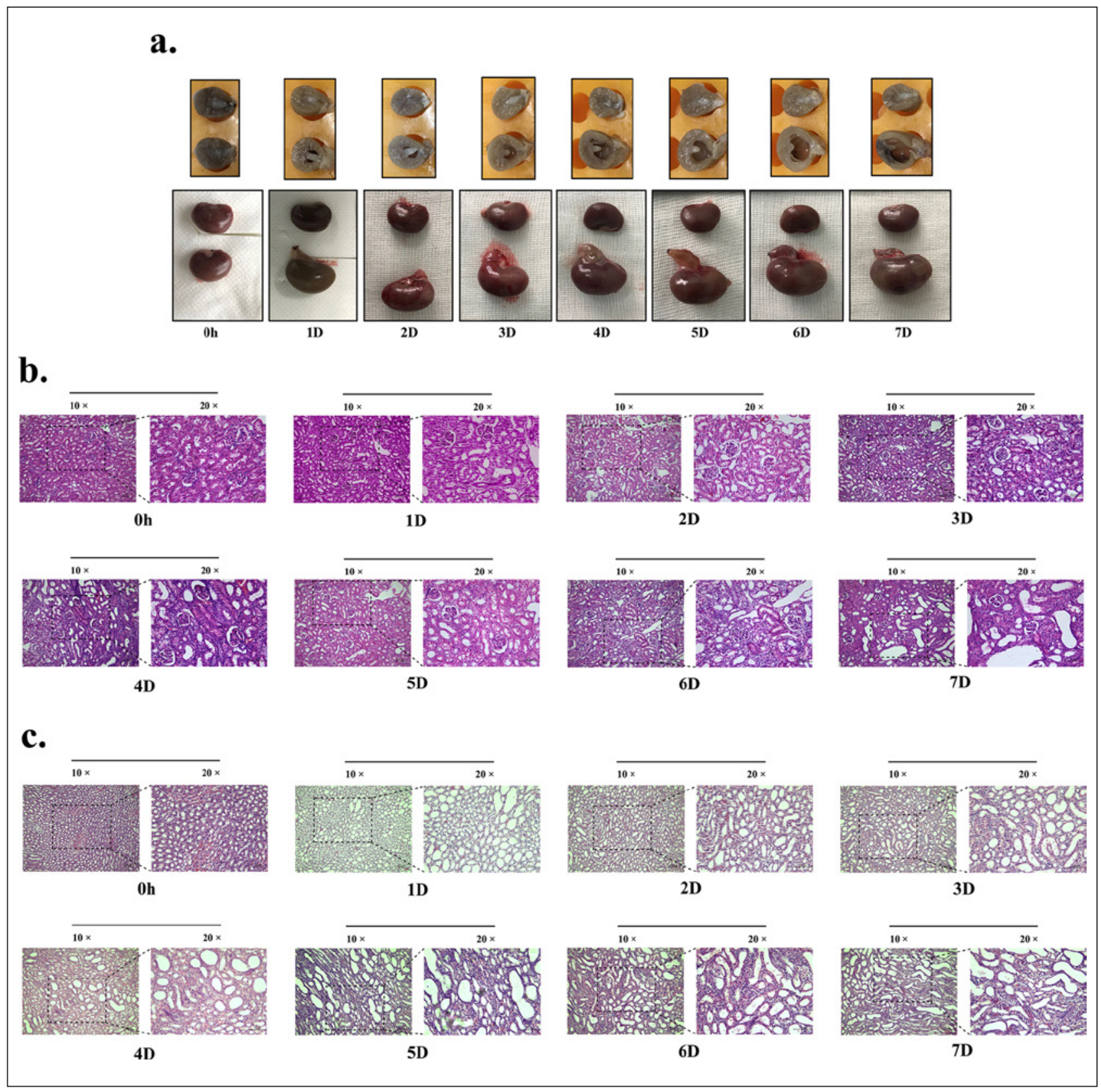

Fig. 2. With the extension of obstruction time, hydronephrosis was aggravated, kidney volume increased, and the kidney parenchyma thickness decreased; a The renal volume and degree of hydronephrosis increased with time; b Renal cortical HE staining showed that the renal tubules in the cortex were atrophied with the aggravation of hydronephrosis; c Renal medullary HE staining showed that intramedullary renal interstitial oedema was increased with the aggravation of hydronephrosis.

\section{CysC and the CKD-EPI CysC Equation Better Reflect the Changes in Renal Function}

The means of determining the patients' eGFR using 6 methods are shown in Table 4. We could conclude that the eGFR determined using various equations was associated with the degree of hydronephrosis. In addition, the diagnostic sensitivity of our evaluation by the receiver operating characteristic curve (Table 5) showed that the AUC for CysC detection of 
Table 1. Univariate analyses of patients with different hydronephrosis according to various clinicopathological variables

\begin{tabular}{|c|c|c|c|c|c|c|}
\hline Characteristics & Total & $\begin{array}{l}\text { Hydronephrosis } \\
\text { group, } n(\%)\end{array}$ & $\begin{array}{l}\text { Mild } \\
\text { hydronephrosis } \\
\text { group, } n(\%)\end{array}$ & $\begin{array}{l}\text { Moderate } \\
\text { hydronephrosis } \\
\text { group, } n(\%)\end{array}$ & $\begin{array}{l}\text { Severe } \\
\text { hydronephrosis } \\
\text { group, } n(\%)\end{array}$ & $p$ value \\
\hline Total & 200 & $40(20.0)$ & $110(55.0)$ & $32(16.0)$ & $18(9.0)$ & \\
\hline Age, years & & & & & & 0.099 \\
\hline$<40$ & 33 (16.5) & $7(21.2)$ & 17 (51.5) & $7(21.2)$ & $2(6.1)$ & \\
\hline $40-60$ & $111(55.5)$ & $20(18.0)$ & $69(62.7)$ & $16(14.4)$ & $6(5.4)$ & \\
\hline$>60$ & $56(28.0)$ & $13(23.2)$ & $24(42.9)$ & $9(16.1)$ & $10(17.9)$ & \\
\hline Gender & & & & & & 0.151 \\
\hline Female & $69(34.5)$ & $9(13.0)$ & $43(62.3)$ & 13 (18.8) & $4(5.8)$ & \\
\hline Male & $131(65.5)$ & 31 (23.7) & 67 (51.1) & $19(14.5)$ & 14 (10.7) & \\
\hline Course, day & & & & & & 0.007 \\
\hline$<7$ & $124(62.0)$ & $23(18.5)$ & 79 (63.7) & $13(10.5)$ & $9(7.3)$ & \\
\hline$\geq 7$ & $76(38.0)$ & $17(22.4)$ & $31(40.8)$ & $19(25.0)$ & $9(11.8)$ & \\
\hline Body weight, kg & & & & & & 0.047 \\
\hline$<60$ & 39 (19.5) & $4(10.3)$ & $20(51.3)$ & $13(33.3)$ & $2(5.1)$ & \\
\hline $60-80$ & $133(66.5)$ & $29(21.8)$ & $74(55.6)$ & $16(12.0)$ & 14 (10.5) & \\
\hline$>80$ & $28(14.0)$ & $7(25.0)$ & $16(57.1)$ & 3 (10.7) & $2(7.1)$ & \\
\hline Height, cm & & & & & & 0.539 \\
\hline$<160$ & $38(19.0)$ & $6(15.8)$ & $22(57.9)$ & $8(21.1)$ & $2(5.3)$ & \\
\hline $160-170$ & $93(46.5)$ & 17 (18.3) & $56(60.2)$ & $12(12.9)$ & $8(8.6)$ & \\
\hline$>170$ & $69(34.5)$ & 17 (24.6) & $32(46.4)$ & $12(17.4)$ & $8(11.6)$ & \\
\hline BMI, $\mathrm{kg} / \mathrm{m}^{2}$ & & & & & & 0.218 \\
\hline$<18.5$ & $9(4.5)$ & $2(22.2)$ & $3(33.3)$ & $4(44.4)$ & $0(0.0)$ & \\
\hline $18.5-23.9$ & $72(36.0)$ & $12(16.7)$ & $40(55.6)$ & $11(15.3)$ & $9(12.5)$ & \\
\hline$\geq 24$ & $119(59.5)$ & $26(21.8)$ & 67 (56.3) & $17(14.3)$ & $9(7.6)$ & \\
\hline Primary site & & & & & & 0.593 \\
\hline Right & $93(46.5)$ & 17 (18.3) & $51(54.8)$ & $14(15.1)$ & $11(11.8)$ & \\
\hline Left & $107(53.5)$ & $23(21.5)$ & 59 (55.1) & $18(16.8)$ & $7(6.5)$ & \\
\hline Urine protein & & & & & & 0.040 \\
\hline Negative & 157 (78.5) & $29(18.5)$ & 93 (59.2) & 20 (12.7) & $15(9.6)$ & \\
\hline Positive & $43(21.5)$ & $11(25.6)$ & 17 (39.5) & $12(27.9)$ & $3(7.0)$ & \\
\hline Urine sugar & & & & & & 0.855 \\
\hline Negative & $182(91.0)$ & 37 (20.3) & $101(55.5)$ & $28(15.4)$ & $16(8.8)$ & \\
\hline Positive & $18(9.0)$ & $3(16.7)$ & $9(50.0)$ & $4(22.2)$ & $2(11.1)$ & \\
\hline
\end{tabular}

$p$ value $<0.05$ are shown in bold. BMI, body mass index.

renal function in patients was larger than that of BUN, SCr and SUA, and CysC had higher sensitivity than BUN, SCr and SUA (Fig. 4a). Based on the CysC and SCr equations, the CKD-EPI CysC equation detected a larger AUC and highest sensitivity for renal function (Fig. 4b).

\section{Discussion}

Clinically, the urine above the calculi cannot flow smoothly beyond the obstruction caused by ureteral calculi, and the urine collects in the renal pelvis and calyces. Excessive pressure leads to renal pelvis and calices expansion, renal unit cell degeneration, atrophy and necrosis. Subsequently, this leads to thinning of the renal parenchyma, which increases the hemodynamic resistance of the renal parenchyma and cortical internal arteries, aggravating the degree of hydronephrosis and reducing renal perfusion, thus damaging renal function [23]. 
Kidney

Blood Pressure

Research
Kidney Blood Press Res 2020;45:109-121

Mao et al.: CysC in Ureteral Calculi Hydronephrosis

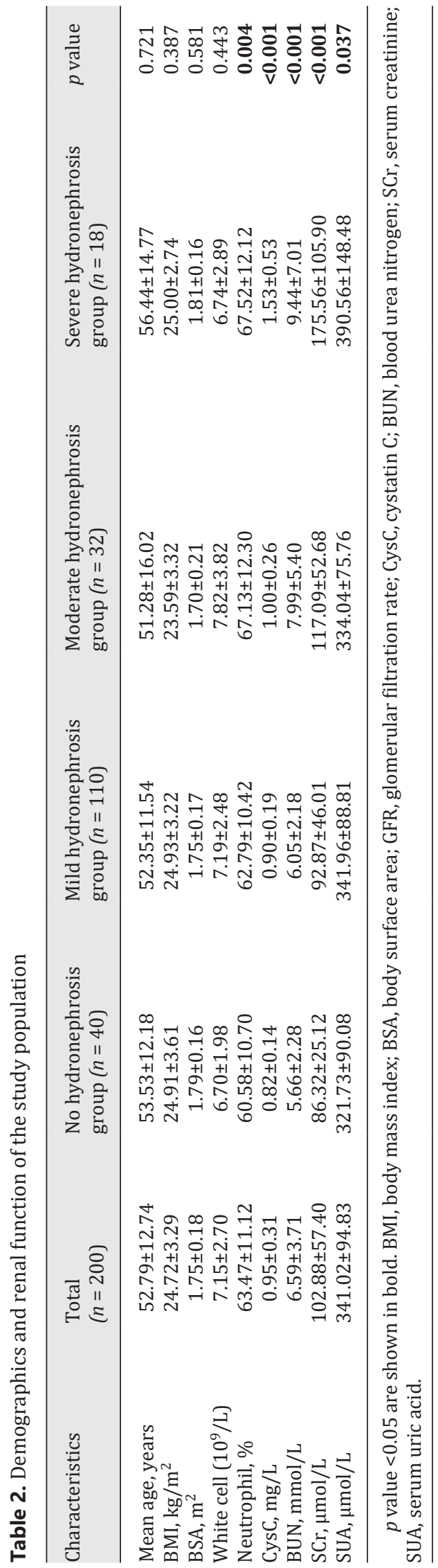

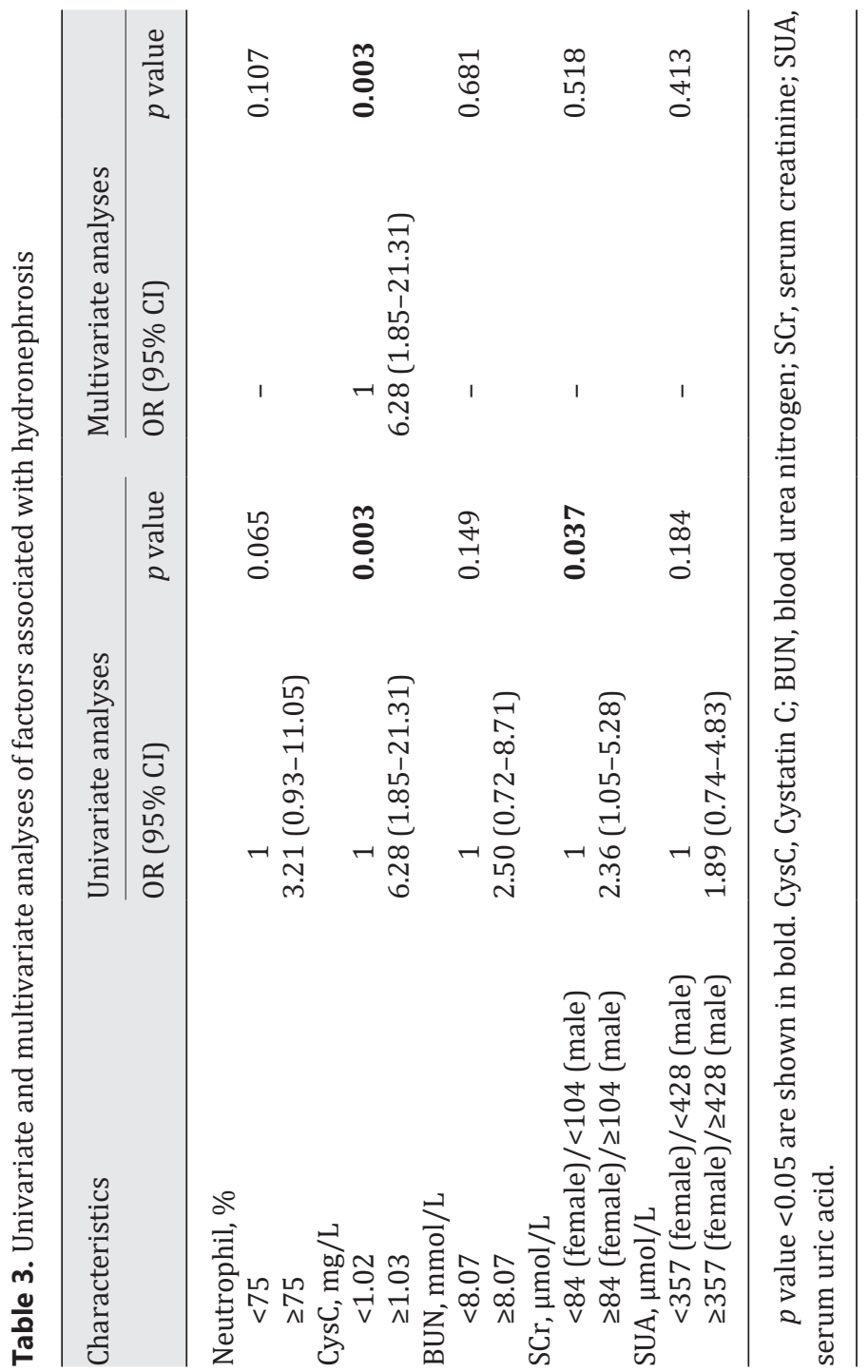


a.

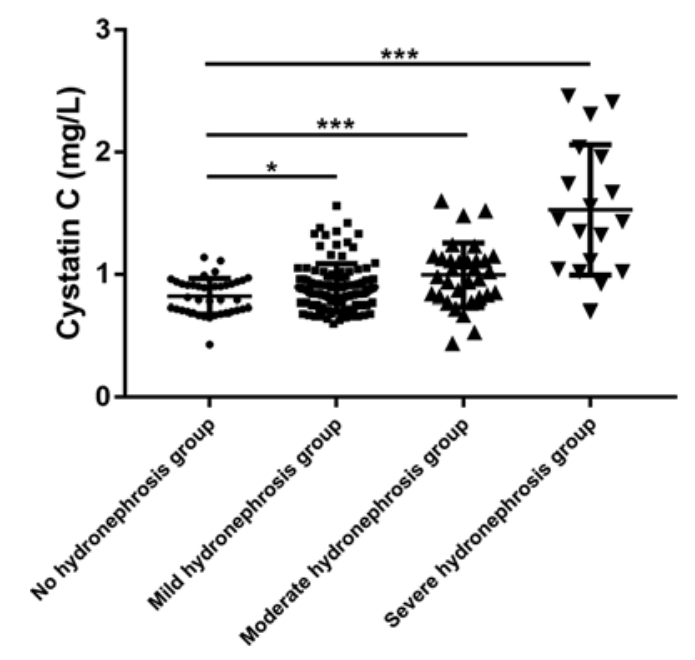

b.

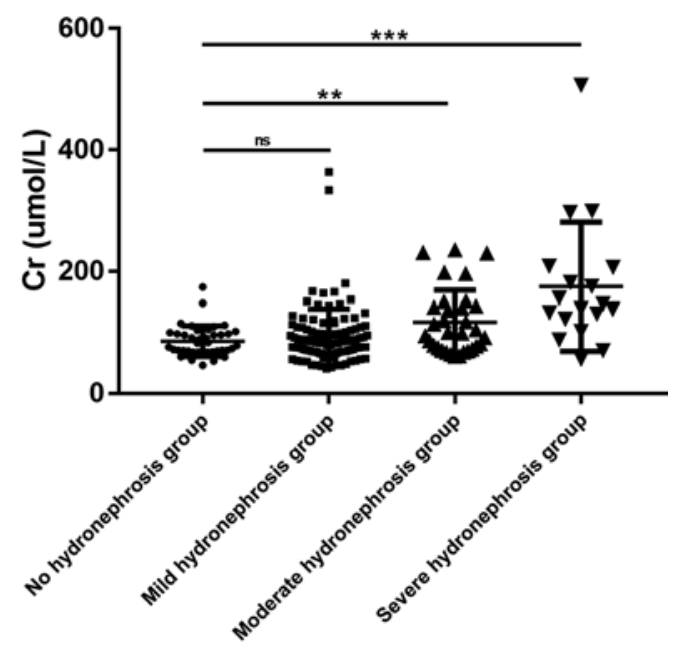

Fig. 3. The relationship between the degree of hydronephrosis and the concentration of CysC (a) and $\mathrm{SCr}$ (b; $\left.n=200,{ }^{*} p<0.05,{ }^{* *} p<0.01,{ }^{* * *} p<0.001\right)$. CysC, Cystatin C; Cr, creatinine.

GFR is a commonly used indicator of renal function changes. Currently, the commonly used indicators include BUN, $\beta_{2}-\mathrm{MG}$, SCr, creatinine clearance, urinary microalbumin, urinary $\mathrm{N}$-acetyl-D-aminoglucosidase and CysC [10, 24, 25]. However, due to the influence of many physiological and pathological factors such as age, sex, body size, and complicated steps of specimen collection, $\mathrm{BUN}, \beta_{2}-\mathrm{MG}, \mathrm{SCr}$, creatinine clearance, urine microalbumin and N-acetyl$\mathrm{D}$-aminoglucosidase are not accurate and reliable in evaluating renal function.

CysC is a low molecular weight protein containing 120 amino acids. It was found that CysC sustain constant velocity in all nuclear cells in vivo as an endogenous GFR marker [12]. CysC can be completely filtered by the glomeruli and do not get degraded and reabsorbed in the renal tubules [26]. Unlike SCr, CysC values are not affected by muscle mass, age, sex and have high specificity making CysC an ideal indicator to evaluate GFR [14].

To improve the accuracy and effectiveness of GFR detection, many specifically designed prediction equations have been proposed as reliable alternatives for evaluating GFR. Commonly used equations are the CG formula, the nephropathy diet improvement equation (MDRD), and the equation for CKD-EPI based on CysC and/or SCr [20-22]. Previously, 84\% of laboratories in United States recommended using the MDRD study equation to estimate GFR $[27,28]$. Studies have found that CKD-EPI equation is a better predictor of risk than MDRD equation in CKD cohort [29], and the CKD-EPI equation is widely used in the North America, Europe, and Australia [30]. However, some studies have shown that MDRD equation has the smallest deviation and the highest accuracy compared with CG formula and CKD-EPI in diabetic cohort [31]. In addition, researchers have also developed new equations that are more accurate than CKD-EPI to eGFR in the elderly in China [32]. This is the first time to explore the expression of $\mathrm{CysC}$ and $\mathrm{SCr}$ in patients with hydronephrosis caused by ureteral calculi.

It has been found that patients with mild, moderate and severe renal hydronephrosis caused by ureteral calculi were all impaired in renal glomerular and renal tubular filtration function [33]. However, for patients with mild and moderate hydronephrosis, SCr may still 
a.

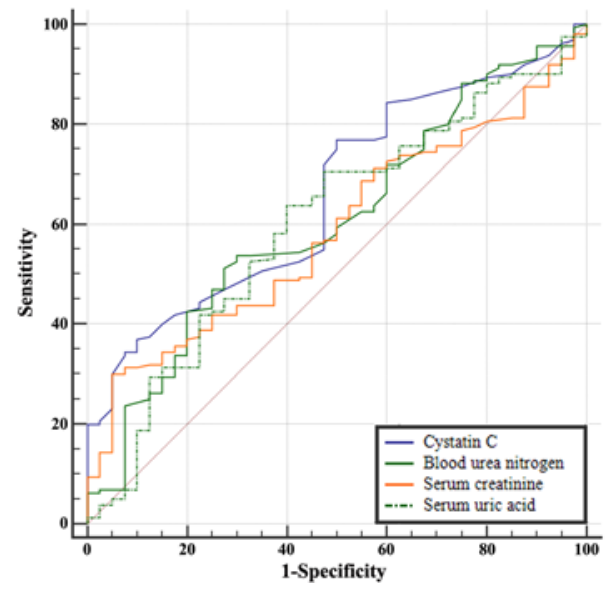

b.

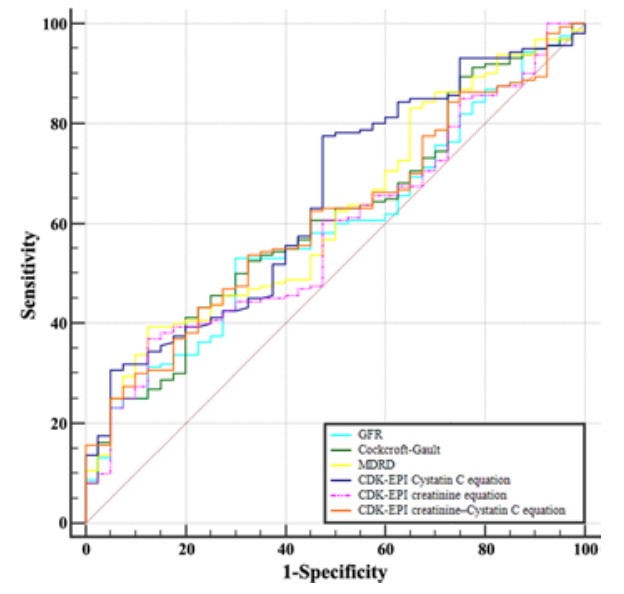

Fig. 4. CysC and the CKD-EPI CysC equation can better reflect the changes in renal function; a ROC curve analysis for CysC, BUN, SCr and SUA; b The ROC curve analysis for the equations based on CysC and SCr. CysC, Cystatin C; BUN, blood urea nitrogen; SCr, serum creatinine; SUA, serum uric acid; GFR, glomerular filtration rate; MDRD, modification of diet in renal disease; CKD-EPI, Chronic Kidney Disease Epidemiology Collaboration.

be in the normal clinical range, but renal function has been impaired. In our study, we judged the degree of hydronephrosis based on the patient's renal ultrasound results [18]. But this is an experienced and professional ultrasound doctor who needs to be involved. Ultrasound doctor with different experiences have different grasps of the degree of hydronephrosis, and the degree of hydronephrosis will be mixed with artificial uncertainty. However, as an indicator in the blood, CysC can be quantified, more convenient and cheaper, and not subject to artificial factors, and CysC as a sensitive indicator can reflect the degree of hydronephrosis.

Our research aims to find a sensitive indicator to reflect the degree of hydronephrosis in patients with ureteral calculi, and then to make an intervention in the patients. By constructing rat models of unilateral ureteral obstruction and statistically analysed various metabolic parameters and clinical variables of patients diagnosed with unilateral ureteral calculi in our hospital, we found that CysC and CKD-EPI CysC equation were more sensitive and accurate for assessing renal function in patients with hydronephrosis caused by ureteral calculi, patients would be more inclined to intervene surgically sooner rather than later compared to a patient in whom no compromise in renal function is detected.

There are several limitations in this study. First, all patients need to be followed up prospectively, and the sample numbers need to further expand. Moreover, approximately $28 \%$ of patients are over the age of 60 , which might have repercussion on physiological renal function decline. Additionally, the underlying etiologic disorders for the development of urinary calculi are multiple, which requires to accurately select patients and identify the aetiology of stone disease.

In conclusion, we found that the expression of $\mathrm{Cys} C$ and $\mathrm{SCr}$ increased with the aggravation of hydronephrosis. Moreover, CysC and the CKD-EPI CysC equation can better reflect the changes in renal function in patients with hydronephrosis caused by ureteral calculi. 
Kidney

Blood Pressure

Research
Kidney Blood Press Res 2020;45:109-121

DOI: 10.1159/000504441

www.karger.com/kbr

Mao et al.: CysC in Ureteral Calculi Hydronephrosis
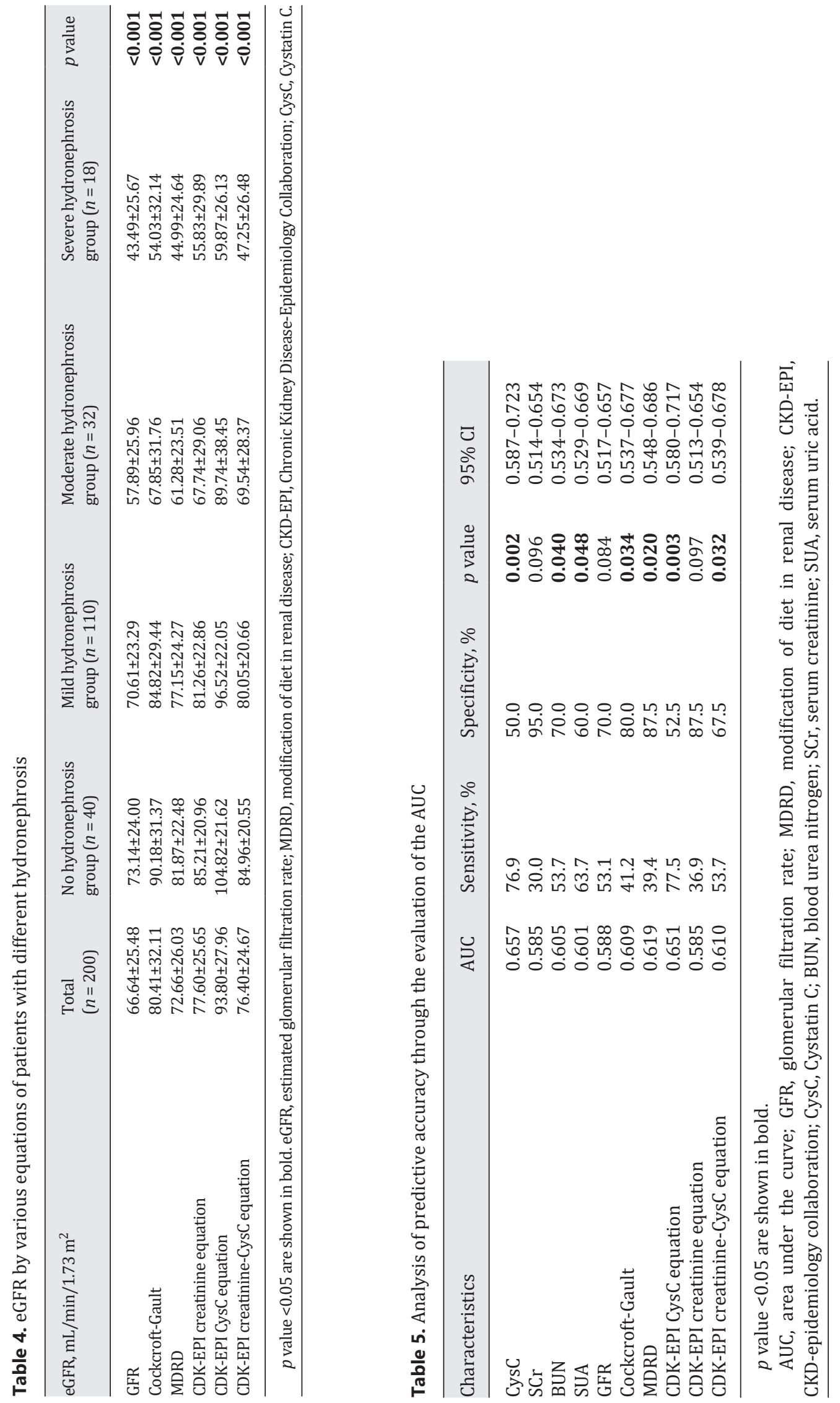


\section{Statement of Ethics}

The study protocol was approved by the Biomedical Ethics Committee of the Tenth Hospital in Shanghai. The study conforms to the ethical standards of the Declaration of Helsinki.

\section{Disclosure Statement}

All authors declare no conflicts of interest.

\section{Funding Source}

This work was supported by grant from the National Natural Science Foundation of China (81001134) to Jiang Geng, Shanghai Science and Technology Commission (Grant No. 18140900302) and the National Natural Science Foundation of China (31670772) to Bo Peng.

\section{Author Contribution}

W.M., S.L., and J.G.: designed the research. K.W., M.W., H.S., Q.L., and M.B.: acquired the data. W.M. and K.W.: analysed the results. W.M., S.L., and K.W.: wrote the article. B.P. and J.G.: revised and provided critical comments. All authors read and approved the final manuscript.

\section{References}

1 Utanğaç MM, Sancaktutar AA, Tepeler A. Micro-ureteroscopy for the treatment of distal ureteral calculi in children. J Pediatr Surg. 2017 Mar;52(3):512-6.

2 Cancian M, Brito J, Renzulli J 2nd, Pareek G. Endourologic and open ureterolithotomy and common sheath reimplant for large bladder and distal ureteral calculi. J Endourol Case Rep. 2016 Nov;2(1):209-11.

3 Dreger NM, Degener S, Ahmad-Nejad P, Wöbker G, Roth S. Urosepsis-etiology, diagnosis, and treatment. Dtsch Arztebl Int. 2015 Dec;112(49):837-47.

4 Wang CJ, Hsu CS, Chen HW, Chang CH, Tsai PC. Percutaneous nephrostomy versus ureteroscopic management of sepsis associated with ureteral stone impaction: a randomized controlled trial. Urolithiasis. 2016 Oct; 44(5): 415-9.

5 Krzemień G, Szmigielska A, Bombiński P, Barczuk M, Biejat A, Warchoł S, et al. Extreme hydronephrosis due to uretropelvic junction obstruction in infant (case report). Dev Period Med. 2016 Apr-Jun;20(2):139-42.

6 Cox C, MacDonald S, Henneberry R, Atkinson PR. My patient has abdominal and flank pain: identifying renal causes. Ultrasound. 2015 Nov;23(4):242-50.

7 Janeczko M, Niedzielska E, Pietras W. Evaluation of renal function in pediatric patients after treatment for wilms' tumor. Adv Clin Exp Med. 2015 May-Jun;24(3):497-504.

8 Henriksen UL, Henriksen JH. The clearance concept with special reference to determination of glomerular filtration rate in patients with fluid retention. Clin Physiol Funct Imaging. 2015 Jan;35(1):7-16.

9 Pottel H, Hoste L, Dubourg L, Ebert N, Schaeffner E, Eriksen BO, et al. An estimated glomerular filtration rate equation for the full age spectrum. Nephrol Dial Transplant. 2016 May;31(5):798-806.

10 Zhou SM, Zhao W, Lin T, Zhao ST, Wang HW. Serum Cystatin C can detect impaired graft function early after renal transplantation. Int J Clin Exp Med. 2015 Sep;8(9):16379-83.

11 Aksun SA, Ozmen D, Ozmen B, Parildar Z, Mutaf I, Turgan N, et al. Beta2-microglobulin and cystatin c in type 2 diabetes: Assessment of diabetic nephropathy. Exp Clin Endocrinol Diabetes. 2004 Apr;112(4):195-200.

12 Abrahamson M, Grubb A, Olafsson I, Lundwall A. Molecular cloning and sequence analysis of cDNA coding for the precursor of the human cysteine proteinase inhibitor cystatin C. FEBS Lett. 1987 Jun;216(2):229-33.

13 Filler G, Bökenkamp A, Hofmann W, Le Bricon T, Martínez-Brú C, Grubb A. Cystatin C as a marker of GFRhistory, indications, and future research. Clin Biochem. 2005 Jan;38(1):1-8.

14 Liu J. Evaluation of serum cystatin $C$ for diagnosis of acute rejection after renal transplantation. Transplant Proc. 2012 Jun;44(5):1250-3. 
15 Otsuka T, Tanaka A, Suemaru K, Inoue T, Nishimiya T, Murase M, et al. Evaluation of the clinical application of cystatin $\mathrm{C}$, a new marker of the glomerular filtration rate, for the initial dose-setting of arbekacin. J Clin Pharm Ther. 2008 Jun;33(3):227-35.

16 Parasuraman S, Raveendran R, Kesavan R. Blood sample collection in small laboratory animals. J Pharmacol Pharmacother. 2010 Jul;1(2):87-93.

17 Mao W, Huang X, Wang L, Zhang Z, Liu M, Li Y, et al. Circular RNA hsa_circ_0068871 regulates FGFR3 expression and activates STAT3 by targeting miR-181a-5p to promote bladder cancer progression. J Exp Clin Cancer Res. 2019 Apr;38(1):169.

18 Noble VE, Brown DF. Renal ultrasound. Emerg Med Clin North Am. 2004 Aug;22(3):641-59.

19 Inker LA, Schmid CH, Tighiouart H, Eckfeldt JH, Feldman HI, Greene T, et al.; CKD-EPI Investigators. Estimating glomerular filtration rate from serum creatinine and cystatin C. N Engl J Med. 2012 Jul;367(1):20-9.

20 Levey AS, Stevens LA, Schmid CH, Zhang YL, Castro AF 3rd, Feldman HI, et al.; CKD-EPI (Chronic Kidney Disease Epidemiology Collaboration). A new equation to estimate glomerular filtration rate. Ann Intern Med. 2009 May;150(9):604-12.

21 Cockcroft DW, Gault MH. Prediction of creatinine clearance from serum creatinine. Nephron. 1976;16(1): 31-41.

22 Matsushita K, Mahmoodi BK, Woodward M, Emberson JR, Jafar TH, Jee SH, et al.; Chronic Kidney Disease Prognosis Consortium. Comparison of risk prediction using the CKD-EPI equation and the MDRD study equation for estimated glomerular filtration rate. JAMA. 2012 May;307(18):1941-51.

23 Severgina LO, Leonova LV, Severgina ES, Gurevich AI, Menovshchikova LB, Petrukhina IV, et al. [Coupling between the hemodynamic parameters and the morphological changes in the kidney in children with congenital hydronephrosis]. Arkh Patol. 2011 Mar-Apr;73(2):14-7.

24 Xuan W, Dong M, Dong M. Effects of compound injection of Pyrola rotundifolia L and Astragalus membranaceus Bge on experimental guinea pigs' gentamicin ototoxicity. Ann Otol Rhinol Laryngol. 1995 May; 104(5): 374-80.

25 Tsai HJ, Chen BH, Wu CF, Wang SL, Huang PC, Tsai YC, et al. Intake of phthalate-tainted foods and microalbuminuria in children: The 2011 Taiwan food scandal. Environ Int. 2016 Apr-May;89-90:129-37.

26 Kimura K, Morita H, Daimon M, Horio M, Kawata T, Nakao T, et al. Utility of cystatin c for estimating glomerular filtration rate in patients with muscular dystrophy. Int Heart J. 2016 May;57(3):386-8.

27 National Kidney Foundation. K/DOQI clinical practice guidelines for chronic kidney disease: evaluation, classification, and stratification. Am J Kidney Dis. 2002 Feb;39(2 Suppl 1):S1-266.

28 Miller WG. Estimating glomerular filtration rate. Clin Chem Lab Med. 2009;47(9):1017-9.

29 Matsushita K, Mahmoodi BK, Woodward M, Emberson JR, Jafar TH, Jee SH, et al. Chronic Kidney Disease Prognosis C: comparison of risk prediction using the ckd-epi equation and the mdrd study equation for estimated glomerular filtration rate. JAMA. 2012;307:1941-51.

30 Earley A, Miskulin D, Lamb EJ, Levey AS, Uhlig K: Estimating equations for glomerular filtration rate in the era of creatinine standardization: A systematic review. Ann Intern Med. 2012 Jun;156(11):785-95.

31 Schwandt A, Denkinger M, Fasching P, Pfeifer M, Wagner C, Weiland J, et al. Comparison of MDRD, CKD-EPI, and Cockcroft-Gault equation in relation to measured glomerular filtration rate among a large cohort with diabetes. J Diabetes Complications. 2017 Sep;31(9):1376-83.

32 Liu X, Wang Y, Wang C, Shi C, Cheng C, Chen J, et al. A new equation to estimate glomerular filtration rate in Chinese elderly population. PLoS One. 2013 Nov;8(11):e79675.

33 Sällström J, Peuckert C, Gao X, Larsson E, Nilsson A, Jensen BL, et al. Impaired EphA4 signaling leads to congenital hydronephrosis, renal injury, and hypertension. Am J Physiol Renal Physiol. 2013 Jul;305(1):F71-9. 